\title{
A critical review of the assessment of Vitamin D
} \section{status}

\begin{abstract}
Vitamin D sufficiency, or lack thereof, has become an area of increased clinical interest. Testing for vitamin D status using the serum 25 hydroxycalciferol assay, as is frequently done, is costly and the benefit of such testing is unclear. Inappropriate use of this test procedure frequently leads to inappropriate treatment with excessive amounts of vitamin $\mathrm{D}$, which can be toxic to the point of life threatening. This scenario is particularly true for disease states, such as osteoporosis, which are not the result of vitamin D deficiency. The goal of this review is to increase the understanding of the physiology of vitamin D metabolism and the implications for the various tests used to assess vitamin D status. This should, in turn, lead to more rational, and reduced, testing and treatment, The result of this would be anticipated to include decreased cost, both financial and physical, of bone disease management and reduced risk by better identifying those for whom therapeutic vitamin D supplementation is actually indicated.
\end{abstract}

Volume 6 Issue 3 - 2018

\author{
Arthur B Chausmer \\ Division of Endocrinology and Metabolism, Johns Hopkins \\ University School of Medicine, USA
}

\author{
Correspondence: Arthur B Chausmer, MD PhD, F.A.C.P. \\ F.A.C.E., F.A.C.N., C.N.S., Adjunct Professor of Medicine, \\ Division of Endocrinology and Metabolism \\ Johns Hopkins University School of Medicine, 7I I I Piney \\ Woods PI, Laurel, MD 20707, USA, Email AChausmer@ca-info. \\ com
}

Received: May 18, 2018 | Published: June 27, 2018

\section{Introduction}

In the United States the evaluation of a patient with osteoporosis frequently includes an assessment of vitamin D status. In this review we will examine the role of vitamin $\mathrm{D}$ in bone and calcium metabolism in general as well as osteoporosis and how best to assess its sufficiency. In general practice, this is most frequently done by evaluation of the serum 25 hydroxycalciferol $(25(\mathrm{OH}) \mathrm{D})$ level, an intermediate in the vitamin D pathways. The reasons for this are not entirely clear and will be critically considered.

Vitamin D is not a single monolithic entity. In general there are two basic molecules in common use, ergocalciferol, vitamin D2, and cholecalciferol, vitamin D3. Ergocalciferol is a plant sterol which can act as a surrogate for the animal form, cholecalciferol. It is most often ingested as a dietary supplemental source of vitamin D. When dietary vitamin D intake is considered, they are essentially interchangeable. Therefore, the current assessment will deal only with cholecalciferol.

Because vitamin D is not a monolithic entity and is frequently used imprecisely to refer to one or the other of the intermediates, the term "vitamin D" will be used when it refers generally and not to any specific intermediate. It is also important to note here that this discussion is limited to adults. Juvenile vitamin D deficiency, rickets unassociated with genetic abnormalities of phosphorus metabolism or specific enzyme pathways, will not be discussed at any length here.

\section{Review of the intermediary pathways}

Vitamin D might best be called a hormone rather than a vitamin in that it is synthesized in one part of the body, the skin, secreted directly into the blood where it is bound to a specific transport protein, $\mathrm{D}$ binding protein (DBP, VDBP), and transported to other tissues. There are intermediate conversion steps before it is transported by the same DBP to the end organs when it exerts it's biologically important actions. Unlike other hormones, however, there are exogenous nutritional sources which feed directly into this activation pathway.

The DBP in its several forms provides another level of regulation and may be important since both the binding affinities of the various D metabolites and the amount bound versus free can significantly alter the kinetics and bioavailability of the substrate in the activation pathways for vitamin $\mathrm{D}$. This is unaccounted for in most estimations of vitamin D status. Curiously, it appears more is known about non classical vitamin $\mathrm{D}$ effects than with its role in the $\mathrm{D}$ regulatory processes. Because of the very limited amount of information available and the lack of definitive information about its interactions in the pathways beyond acting as a transport medium, apart from noting its potential importance, it will not be further addressed in this review. ${ }^{1-6}$

The vitamin D metabolic precursor is first synthesized in the skin from cholesterol to 7 dehydrocholesterol and then under the influence of UV-B ultraviolet light ( 290 to $310 \mathrm{~nm}$ ) to lumisterol and tachysterol and ultimately to cholecalciferol. ${ }^{7,8}$ This is also the substance which is nutritionally available from dietary sources. From this point the paths are identical for the endogenous and exogenous intermediate, cholecalciferol. The binding protein, DBP, acts as a transport medium to bring the cholecalciferol to the liver where it taken up and acted upon by 25 hydroxylases, a number of cytochrome $\mathrm{P} 450$ enzymes, to form the 25 hydroxycholecalciferol $(25(\mathrm{OH}) \mathrm{D}, 25(\mathrm{OH})$ calciferol, or calcidiol). Although there are other tissues which also contain these enzymes, they play a minor role in this conversion step. While this is the first actual hydroxylation step, the molecule is now a diol since the cholecalciferol already contains one hydroxyl group as part of the native molecule and the first hydroxylation step provides a second $\mathrm{OH}$ group. This first hydroxylation step occurs fairly quickly, but the entire process takes a variable, but relatively long, period of time while the calciferol or 25 OHD metabolite are stored in the liver. The precise kinetics of this is unknown. The product, 25(OHD, is an essentially inactive intermediate metabolite with no proven biological activity. It is also the metabolite most often measured as an assessment of "vitamin D" status.

The 25 hydroxycholecalciferol is released from the liver and the fat mass and bound to the same transport protein, DBP, where it is taken up by the kidney and acted on by a 1 alpha hydroxylase or 24 hydroxylase to form either 1,25 dihydroxycholecalciferol $(1,25(\mathrm{OH}) 2$ D) or calcitriol since it now has a total of 3 hydroxyl groups or 24,25 dihydroxycholecalciferol $(24,25(\mathrm{OH}) 2 \mathrm{D})$. The 24,25 metabolite is inactive. The 1 alpha (CYP25B1) is an inner mitochondrial P-450 mixed function oxidase located primarily in the cells of the proximal renal tubule. ${ }^{9}$ The $1,25(\mathrm{OH}) 2 \mathrm{D}$ is the only proven active metabolite 
in the pathway. While the transit time from $25(\mathrm{OH}) \mathrm{D}$ pool to 1,25 $(\mathrm{OH}) 2 \mathrm{D}$ pool is not definitively known, it appears fairly short and it appears that the limiting factor in the production of the 1,25 metabolite may be, at least in part, availability of the the 25 OHD precursor. Other tissues, such as the placenta and macrophages, have also been demonstrated to have the 1 alpha hydroxylase, but it is the kidney which, under normal circumstances, is the primary site of activation of the $25(\mathrm{OH}) \mathrm{D} .{ }^{10}$ There is also some evidence supporting the presence of intraparathyroid 1 alpha hydroxylase. This is functionally similar, but not identical, to renal 1 alpha hydroxylase and it is probable that this exerts an autocrine/paracrine regulation of parathyroid hormone synthesis. ${ }^{11}$

It is also important to remember that all of the vitamin D metabolites are fat soluble and the fat mass is a storage site for these metabolites. Since it is not known with any accuracy how much is in the fat mass at any time, storage and availability assessments are negatively impacted. Storage and release assessments depend not only on availability, but also the size of the fat mass and the undefined factors regulating movement into and out of the fat mass. Related to this, as a fat soluble vitamin, any surgery or disease process which alters fat absorption may affect vitamin D status and any evaluation of overall D status should be adjusted to account for this fat malabsorption, possible changes in transport kinetics, as well as the total fat mass and other possible contributing influences.

\section{Review of the regulation of vitamin $D$ and calcium homeostasis}

In order to assess the appropriateness of any testing procedure, an understanding of the regulation of the intermediate steps in the activation process is critical to the overall understanding. Of vitamin $\mathrm{D}$ homeostasis. It is beyond the scope of this paper to provide a complete review of calcium homeostasis, only relatively cursory references will be addressed here, but it is important to understand that these regulatory inter relationships play a central role in the understanding of the whole. They mutually and complexly regulate each other. All of the processes in the regulation of calcium homeostasis are inextricably linked to those in the vitamin D metabolic pathways and actions.

Clearly the first step in the pathway is at the synthesis step or at the dietary step. If there is an inadequate exposure of appropriate ultraviolet radiation (UV-B) to the skin, sufficient amounts of cholecalciferol cannot be synthesized. This can be the result of natural events, such as living in areas with limited sunlight and/or where the environment requires heavy protective clothing even when the sun may be available. Such seasonal conditions become more significant as one moves the farther from the equator with the geographic factor being most severe near the north or south poles. ${ }^{12}$ Another natural event is the evolution of race. Races which have evolved to survive in the north are better at making calciferol than those which evolved nearer the equator which are high sunlight areas and thus have more protective melanin skin pigmentation which absorbs UV radiation and therefore less calciferol synthesis. ${ }^{9}$ Where a light skinned caucasian may require only 15 or 20 minutes of sun exposure to synthesize enough calciferol to meet daily needs, a dark skinned black may require 4 or 5 times that amount.

Culture may also play a role. While less common now, some cultures kept women and children inside or near completely covered with clothing when outside. Even men who wore broad brimmed hats and spent most of the day inside were susceptible to inadequate calciferol production. Culture may also be reflected in diet in that diets with inadequate calciferol or ergocalciferol diets could also result in significant deficiency. Full vegans may be at risk since dietary cholecalciferol is animal in origin and, unless taken as a supplement, diet can generally not provide adequate ergocalciferol. ${ }^{13}$ In the United States, at least, milk is a major source of vitamin D because it is fortified with vitamin D. "Natural" milk contains very little if any vitamin $\mathrm{D}$ precursor. For peoples who live in the far north where there is less sunlight, animal liver is common in the diet and provides a good dietary source for cholecalciferol.

Production of the $25 \mathrm{OHD}$ is dependent on both the presence of substrate, cholecalciferol, and the activity of the 25 hydroxylase. The most common modifier of the hepatic 25 hydroxylase is pharmacologic, particularly anti seizure medications which interfere with the cytochrome P450 vitamin D 25 hydroxylases, primarily CYP2R1, but also including CYP2D11 and CYP2D25. Several drugs, particularly the anti seizure drugs, can interfere with these enzyme systems, particularly phenobarbital, although any drug which interferes with the the cytochrome P450s can potentially have deleterious effects. ${ }^{14,15}$ Interestingly, several other anti convulsives, which do not appear to interact with the cytochrome system have also been reported to interfere with 25 hydroxylase through unknown mechanisms. Genetic factors can also affect this hydroxylation. A patient with a homozygous mutation of the CYP2R1 gene was found with low circulating levels of 25(OH)D3 and classic symptoms of vitamin $\mathrm{D}$ deficiency. ${ }^{16}$ Although this must be considered a very rare cause, it does suggest that other, partial; alterations of the enzyme can affect this step in the pathway. Nevertheless, the pool sizes of the hepatic and extra hepatic 25 OHD pools and the major physiologic regulators of the 25 hydroxylation of calciferol remain essentially unknown.

The $25(\mathrm{OH}) \mathrm{D}$ is transported to the kidney by the DBP when it is further hydroxylated to either the $1,25(\mathrm{OH}) 2 \mathrm{D}$ or the 24,25 $(\mathrm{OH}) 2 \mathrm{D}$ under the influence primarily of the serum phosphorus concentration. Low phosphate favors 1 alpha hydroxylation and high phosphate favors the synthesis of $24,25(\mathrm{OH}) 2 \mathrm{D}$. The low phosphorus would then act to increase the active 1,25(OH)2D with a resultant increase in gastrointestinal calcium absorption and, as a result, the serum calcium. This, in turn, reduces PTH which would then conserve phosphorus and negatively feedback regulates the 1 alpha hydroxylations. This is, however, a very complex feedback mechanism. The initiating factor is frequently hypocalcemia which would result in parathyroid hormone release, presumably as a result of the low $\mathrm{Ca}$, which would result in some hypophosphatemia, also because PTH causes renal phosphaturia. The resultant decrease in serum phosphorus would then stimulate 1 alpha hydroxylation. The increased 1,25(OH)2D would increase gastrointestinal absorption of calcium and reduce the hypocalcemia. The increase in PTH would also result in amelioration of the hypocalcemia by release of calcium from bone stores which would also be a feedback regulatory mechanism as well as the increased calcium absorption from the GI tract. Clearly PTH indirectly, and possibly directly, is a key regulator of vitamin D homeostasis. Thus, PTH needs to be considered when assessing vitamin D status.

Other pathologic states can also have unexpected effects on vitamin $\mathrm{D}$ and calcium metabolism. While in ESRD the problem is too little active $1,25(\mathrm{OH})_{2} \mathrm{D}$, in sarcoidosis and some other granulomatous diseases, including tuberculosis, the tissue contains sufficient 1 alpha 
hydroxylase to cause clinically significant hypervitaminosis D and hypercalcemia. ${ }^{17,18}$

Fibroblast growth factor 23, FGF23, has also emerged as a regulating factor for 1 alpha hydroxylation. FGF 23 is a phosphaturic factor secreted by bone that promotes renal phosphate excretion by decreasing its reabsorption in the proximal tubule, and is also a physiological regulator of vitamin $\mathrm{D}$ metabolism by much the same mechanism as PTH noted above as well as by direct inhibition of $1,25(\mathrm{OH})^{2} \mathrm{D}$ biosynthesis. ${ }^{19,20} 1,25(\mathrm{OH}) 2 \mathrm{D}$ stimulates FGF 23 in bone and hence functions as a feedback mechanism by inhibiting 1 alpha hydroxylation and stimulating 24 hydroxylation. FGF 23 has also been shown to stimulate intra parathyroid 1 alpha hydroxylase with the resultant $1,25(\mathrm{OH}) \mathrm{D}$ inhibiting PTH synthesis. Clinically states in which there is overproduction of FGF 23 result in symptoms of D deficiency. Genetic disorders including autosomal dominant hypophosphatemic vitamin D resistant rickets, $\mathrm{X}$ linked hypophosphatemic rickets and tumor-induced osteomalacia. Hyperphosphatemic tumoral calcinosis has also been shown to be related to unregulated hyperactivity of the 1 alpha hydroxylase resulting in hyperabsorption of calcium and deposition of ectopic calcium deposits. ${ }^{21}$

Finally, other calcium related hormones, particularly calcitonin and prolactin, have been suggested as possible regulators of 1 alpha hydroxylation and therefore may influence regulation in the vitamin D pathway. They are, however, considered to be minor players in the overall regulation of both $\mathrm{D}$ and calcium homeostasis. Calcitonin, which acts to lower calcium in other species but has an undefined role in humans, has been reported to stimulate 1 alpha hydroxylation. The stimulation of $1,25(\mathrm{OH}) \mathrm{D}$ production by calcitonin may have physiological significance during lactation when calcitonin levels as well as $1,25(\mathrm{OH}) \mathrm{D}$ levels are elevated and when the need for calcium is increased. Nonetheless, whatever role calcitonin may have in the physiologic regulation of 1 alpha hydroxylation, not generally thought to be a major one..$^{22-26}$ Much the same can be said for prolactin. It has been suggested that prolactin, which is also elevated during lactation, can stimulate $1,25(\mathrm{OH}) \mathrm{D}$ production. However, this can be managed by bromocriptine or similar drugs, which inhibit pituitary prolactin secretion, and has been reported to significantly reduce plasma $1,25(\mathrm{OH}) \mathrm{D}$ levels in lactating animals. Recent studies have shown that prolactin also has a direct effect on the transcription of the $1 \alpha$ lpha hydroxylase gene. ${ }^{27,28}$

\section{Assessment of vitamin D status}

This brings us to the core question of how best to assess vitamin D status when physiologic principles are considered. These data make it apparent that the popular interpretation of low 25 OHD reflecting vitamin $\mathrm{D}$ deficiencies is incorrect. The arguments for its use have been made that based primarily on opinion supported by correlational indirect data regarding $\mathrm{PTH}$, which has a multiplicity of regulators. ${ }^{29}$

Apart from the fact that $25(\mathrm{OH}) \mathrm{D}$ is an inactive intermediary, there are no data supporting that it is a measure of whole body vitamin D stores of any metabolite since these have never been quantified. Ultimately, if you don't know the pool size, you can't know how full the pool may be. Perhaps more importantly, the pool contents in this case reflect an inactive intermediate. This acceptance of the $25(\mathrm{OH})$ $\mathrm{D}$ as some index of vitamin $\mathrm{D}$ has apparently been based primarily on opinion which has been repeated over and over, one citation using another as the source material in a circular logic, in the absence of any objective proof. The popular wisdom on which the basic assumptions are based rest on correlational studies, most often with PTH as an indirect indicator of calcium status, and on inference, neither of which can be considered definitive. The total body stores of $25(\mathrm{OH})$ $\mathrm{D}$ in humans, or any other mammal for that matter, are unknown the $25(\mathrm{OH}) \mathrm{D}$ making it an unreliable marker of any aspect of vitamin $\mathrm{D}$ status. These are the static assessments. Even if the total body stores of $25(\mathrm{OH}) \mathrm{D}$ were known, the factors that influence movement into or out of those stores are unknown. Since we do not know the flows into or out of that container, again nothing can be said about the status of the dynamics. It is clear that blood levels of an intermediate are never necessarily related to the levels of the end product. Vitamin D status, whatever that is, cannot be accurately and reliably accessed via an intermediate. Thus, nothing can be said about the actual relevance to the total vitamin $\mathrm{D}$ mass nor can anything be said about the relationship of an intermediate to the $1,25(\mathrm{OH}) 2 \mathrm{D}$, which is the active metabolite responsible for all of the known physiologically important effects of vitamin D. Ultimately the $1,25(\mathrm{OH}) 2 \mathrm{D}$ must be measured directly and only that shows sufficiency and clinical status at that point in time, but, like the $25(\mathrm{OH}) \mathrm{D}$, nothing about whole body stores.

There are several examples illustrative of the concepts here. These are but a few of the examples of the concepts described. The blood levels of 25(OH)D are no more an index of whole body D status than serum $\mathrm{Na}$ reflects total body $\mathrm{Na}$ stores in edema or serum $\mathrm{K}$ reflects intracellular $\mathrm{K}$ stores until there is profound depletion. The total body stores of $\mathrm{D}$ can be high, as it is a fat soluble vitamin, and the serum level low if the 25 hydroxylase is relatively inactive which can occur, as we have seen, in a variety of conditions. The stores can be low and the circulating $25(\mathrm{OH}) \mathrm{D}$ high if the 25 hydroxylase has been stimulated or, as in the case of hyperphosphatemic tumoral calcinosis, the 1 alpha hydroxylase is unregulated and the $25(\mathrm{OH}) \mathrm{D}$ is low because of high utilization and removal from the pool and the 1,25(OH)2D high with evidence of $\mathrm{D}$ toxicity, obviously not a deficiency state in the face of low 25(OH)D.

This leaves the question, what is the best indicator of vitamin D status? There is no perfect or complete way to easily assess vitamin D status. The measurement of the physiologically important molecule, $1,25(\mathrm{OH}) 2 \mathrm{D}$, would appear to be the best indication of whether there is physiologically important deficiency at any given point in time. One cannot readily assess if the whole body stores are replete given the current state of knowledge. Since the clinically important problems are related to the 1,25(OH)2 form of calciferol, this appears to be the best approach. Clearly there are other factors involved, parathyroid hormone, FGF 23, and other players, some of which were discussed above, and these should be taken in consideration in evaluation of the whole picture, but they are not of great value in "routine" screening (Figure 1).

All clinical sampling is all done from the plasma pool, which consists of both free and bound metabolite, of which only the free form is biologically important. This is the result of transfer out of the hepatic $25(\mathrm{OH}) \mathrm{D}$ or the renal $1,25(\mathrm{OH}) 2 \mathrm{D}$ pools as well as transfer in and out of the fat mass. Additionally, the influence of the regulatory elements on the transfer coefficients are not quantitatively known, nor is the amount of metabolite in those pools sampled directly and, as a result, no real assessment of the amount of $25(\mathrm{OH}) \mathrm{D}$, nor of the transfer coefficients in or out of the pools, nor the efficiency of transformation from calciferol to $25(\mathrm{OH}) \mathrm{D}$ within the liver can be implied or assessed. Finally, not included in the scale of this diagram 
is the size of the fat mass or the stores of the fat soluble metabolites in the fat mass. The number of variables and degree of uncertainty is the major reason that one cannot consider any single intermediate element, such as the total serum $25(\mathrm{OH}) \mathrm{D}$ concentrations, as a viable estimate of the overall vitamin D status.

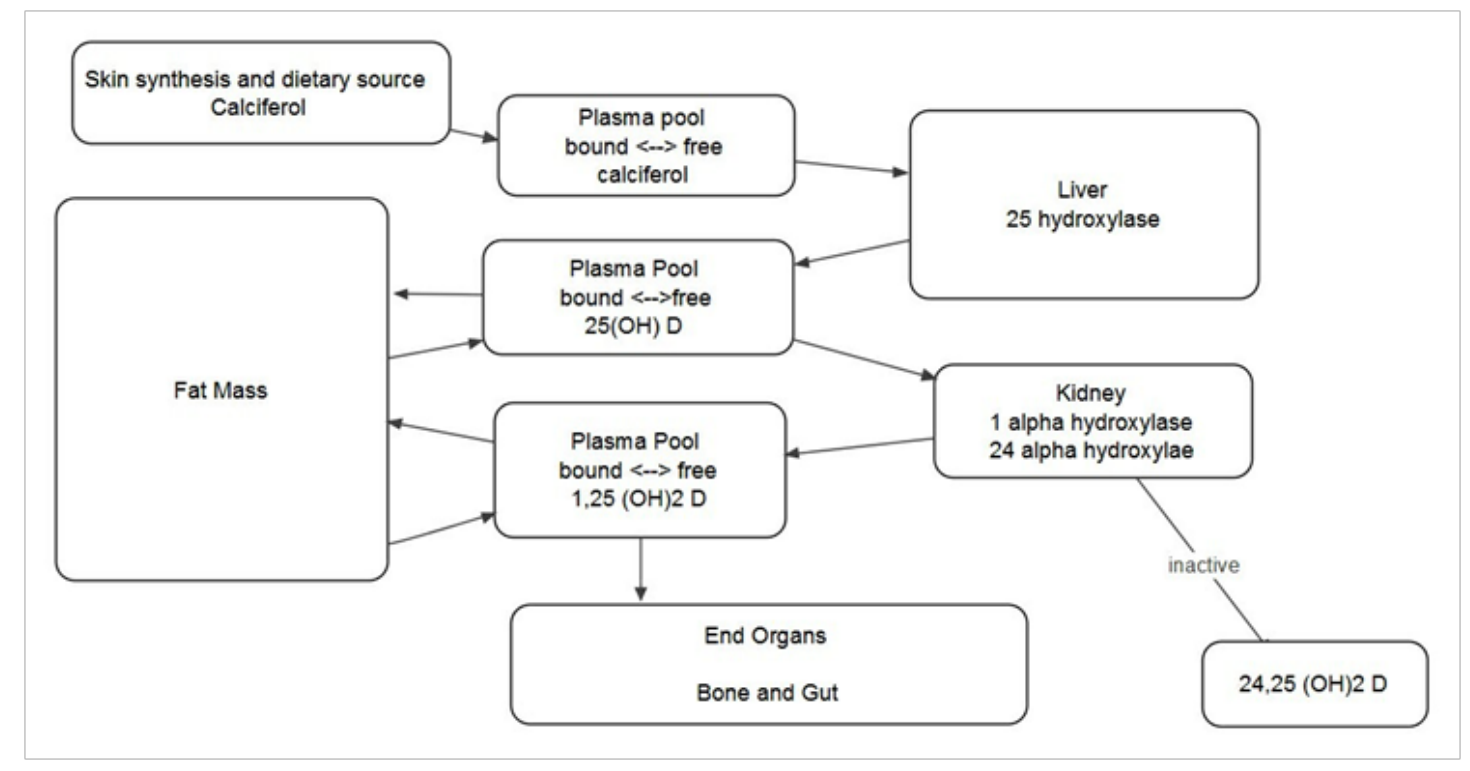

Figure I In this context, it is frequently helpful to visualize this as a compartmental model.

True pure vitamin D deficiency in the general population of adults is vanishingly rare in the United States, the epidemiologic data based on $25(\mathrm{OH}) \mathrm{D}$ screening notwithstanding. True vitamin $\mathrm{D}$ deficiency, is represented clinically in adults only by osteomalacia which can be definitively diagnosed only with undecalcified bone biopsy showing widened osteoid seams or with the classical physical or radiologic signs. It cannot be diagnosed on the basis of the blood concentration of an intermediary. That is not to say that true vitamin D deficiency does not exist. It does in the setting of other problems such as genetic disorders, malabsorption syndromes and the like. Probably the most important cause of true vitamin D deficiency is end stage renal disease (ESRD). ESRD points up many of the physiologic principles at play in vitamin $\mathrm{D}$ homeostasis. There is not enough renal cell mass to convert $25(\mathrm{OH}) \mathrm{D}$ to $1,25(\mathrm{OH}) \mathrm{D}$ and the lack of renal cell mass results in a further inability to excrete phosphorus leading to the characteristic hyperphosphatemia which, in turn, further exacerbates the problem. This leads to hypocalcemia, hyperphosphatemia and secondary hyperparathyroidism resulting in additional bone loss. In this case, for example, the measurement of the $25(\mathrm{OH}) \mathrm{D}$ is useless, even as a measure of whether orally administered vitamin $\mathrm{D}$ is being absorbed and even if it did provide some measure total body D status. Only the $1,25(\mathrm{OH}) \mathrm{D}$ is a valid indicator of vitamin $\mathrm{D}$ status in this situation and only $1,25(\mathrm{OH}) \mathrm{D}$ or an analog can be considered as part of an appropriate treatment regimen.

It is important to note again that the clinical picture of vitamin $\mathrm{D}$ deficiency is osteomalacia, not osteoporosis. Osteomalacia is characterized by poor calcification of osteoid matrix with a disproportion between mineral phase and organic phase of bone. The ratio between the inorganic phases to organic phase of bone is clearly abnormal. Osteoporosis is the result of a mismatch between the kinetic parameters of bone formation and bone resorption with a normal ratio of inorganic to organic phase. The radiographic pictures of osteomalacia and osteoporosis are very different. The bone biopsy pictures of osteomalacia and osteoporosis are very different. To suggest that vitamin D deficiency has a causal role in osteoporosis is to mis interpret the physiologic data. While it is clear that one needs adequate calcium to limit osteoporosis and that there may be some clinical benefit to some supplementation provoking greater gastrointestinal absorption of calcium in osteoporosis, this is not evidence of a vitamin D deficiency state and does not support using pharmacologic, and potentially toxic, doses to treat osteoporosis.

Some important caveats need also to be presented. Pharmacologic doses of calciferol or ergocalciferol can be extremely toxic. While there is some question as to how much is too much, the US Institute of Medicine in its consensus report suggests that 1000 I.U. per day is the maximum recommend dose for most adults in whom dietary supplementation is to be undertaken. ${ }^{30}$ While vitamin D is clearly essential for calcium absorption and may have some benefit in doses of 1000-1500 I.U. per day, more than this may be considered problematic. High dose oral calciferol can overwhelm the normal regulatory mechanisms and provoke severe, life threatening, hypercalcemia. Since it is a fat soluble substance and can remain in the body for prolonged periods, once toxic levels have been reached, the resultant hypercalcemia can be very difficult to treat and result in prolonged hospitalization.

It should also be noted that there are multiple reports suggesting effects of vitamin $\mathrm{D}$ in the broad sense with a variety of other disease states ranging from neurological problems to cardiac problems to baldness. None of these have strong, persuasive support, only suggestive data and nothing in the discussion of the assessment of vitamin D status here should be considered as relating to any these.

It is also important to remember the very basic statistical premise that correlations can never be considered as proof of causality, 
whether in the assessment of vitamin D status or any other situation. Many of the reports suggesting a relationship between 25(OH)D and pathological states rest on these epidemiological correlation studies with secondary or tertiary end points or on in vitro studies, which again may provide suggestive evidence but can never be considered definitive. Meta analytic studies, which are frequently cited as support, also have severe limitations which are beyond the scope of this presentation to review. This all point up the problem that, when considering "evidence based" clinical recommendations, the quality rather than the quantity of that evidence must be considered, and this can be a daunting task, which is particularly true for this topic. Another by product of this "evidence based" phenomenon is that one report may quote another as "evidence", which is in turn quoting another based on non definitive opinion. This may occur through much iteration and becomes an exercise in very circular reasoning which is very difficult to resolve. All of this complicates the resolution of the question as to how best to assess vitamin D status.

\section{Summary}

The accurate assessment of vitamin D overall status requires an understanding of the physiology and biochemistry of the intermediate pathways and regulatory mechanisms as well as the underlying pathology. No element of vitamin D metabolism can be considered in isolation from the larger calcium homeostasis. While vitamin D is essential for calcium absorption and may have some benefit in doses of 1000-1500 iuper day, more than this may be considered problematic. As in most of medicine, there is no simple answer. In general, the serum $25(\mathrm{OH}) \mathrm{D}$ concentration is an inappropriate measurement to assess vitamin D status under virtually any circumstance, although it may be considered with other physiologic data. Only measurement of the $1,25(\mathrm{OH}) 2 \mathrm{D}$ reflects the physiologic vitamin $\mathrm{D}$ status at any given moment in time and there is no index of the status of the storage for any of the vitamin $\mathrm{D}$ metabolites. The definitive diagnosis of pathologically important dietary $\mathrm{D}$ deficiency requires either characteristic $\mathrm{X}$ ray evidence or bone biopsy with undecalcified sections showing widened osteoid seams in the appropriate overall clinical setting.

\section{Acknowledgments}

None.

\section{Disclosure}

The author has no conflicts of interest or financial interests. The views presented here are solely those of the author and not necessarily those of any affiliates.

\section{References}

1. Brown AJ, Coyne DW. Bioavailable vitamin D in chronic kidney disease. Kidney Int. 2012;82(1):5-7.

2. Falleti E, Bitetto D, Fabris C, et al. Vitamin D binding protein gene polymorphisms and baseline vitamin D levels as predictors of antiviral response in chronic hepatitis C. Hepatology. 2012;56(5):1641-50.

3. Zhang J, Habiel DM, Ramadass M, et al. Identification of two distinct cell binding sequences in the vitamin D binding protein. Biochim Biophys Acta. 2010;1803(5):623-9.

4. Fang Y, van Meurs JB, Arp P, et al. Vitamin D binding protein genotype and osteoporosis. Calcif Tissue Int. 2009;85(2):85-93.

5. Nagasawa H, Uto Y, Sasaki H, et al. Gc protein (vitamin D-binding protein): Gc genotyping and GcMAF precursor activity. Anticancer Res. 2005;25(6A):3689-95.

6. Head JF, Swamy N, Ray R. Crystal structure of the complex between actin and human vitamin D-binding protein at 2.5 A resolution. Biochemistry. 2002;41(29):9015-20.

7. Holick M. Factors that influence the cutaneous photosynthesis of pre vitamin D3. Science. 1981;211:590-593.

8. Nair-Shalliker V, Clements M, Fenech M, et al. Personal Sun Exposure and Serum 25-hydroxy Vitamin D Concentrations. Photochem Photobiol. 2012;10:1051-1097.

9. Bikle, D, Adams, J, Christakos S. Vitamin D production, metabolism, mechanism of action and clinical requirements. 2008: Primer on Metabolic Bone Disease Seventh edition, Amer Soc for Bone and Mineral Research. 2013.

10. Christakos S, Ajibade DV, Dhawan P, et al. Vitamin D: Metabolism Endocrinol Metab Clin North Am. 2010;39(2):243-53.

11. Ritter CS, Haughey BH, Armbrecht HJ, et al. Distribution and regulation of the 25- hydroxyvitamin D3 $1 \alpha$-hydroxylase in human parathyroid glands. Steroid Biochem Mol Biol. 2012:130(1-2):73-80.

12. Webb A, Kline L, Holick M. Influence of season and latitude on the cutaneous syntheses of vitamin D3. J Clin Endocr Metab. 1988;67(2):373-8.

13. Walsh S. Vegans and the Sunshine vitamin. The Vegan. 2008. 6-7 p.

14. Meier C, Kraenzlin ME. Antiepileptics and bone health. Ther Adv Musculoskeletal Dis. 2011;3(5):235-43.

15. Verrotti A. Bone and calcium metabolism and antiepileptic drugs. Clin Neurol Neurosurg. 2010;112(1):1-10.

16. Cheng JB, Levine MA, Bell NH, et al. Genetic evidence that the human CYP2R1 enzyme is a key vitamin D 25-hydroxylase. Proc Natl Acad Sci US A. 2004;101(20):7711-5.

17. Wallach S. Personal Communication.

18. Jacob, TP, Bilzikian JP. Rare Causes of Hypercalcemia. J Clin Endo Metab. 2005;90(11):6316.

19. Bai X, Miao D, Li J, et al. Transgenic mice overexpressing human fibroblast growth factor 23 (R176Q) delineate a putative role for parathyroid hormone in renal phosphate wasting disorders. Endocrinology. 2004;145:5269-79.

20. Weber TJ, Liu S, Indridason OS, et al. Serum FGF23 levels in normal and disordered phosphorus homeostasis. J Bone Miner Res. 2003;18(7):1227-34

21. Prince MJ, Schaeffer PC, Goldsmith RS, et al. Hyperphosphatemic tumoral calcinosis: association with elevation of serum 1,25-dihydroxycholecalciferol concentrations. Ann Intern Med. 1982;96(5):586-91.

22. Shinki T, Ueno Y, DeLuca HF, et al. Calcitonin is a major regulator for the expression of renal 25-hydroxyvitamin D3-1alpha-hydroxylase gene in normocalcemic rats. Proc Natl Acad Sci U S A. 1999:96(14):8253-8.

23. Kawashima H, Torikai S, Kurokawa K. Calcitonin selectively stimulates 25-hydroxyvitamin D3-1 alpha-hydroxylase in proximal straight tubule of rat kidney. Nature. 1981;291(5813):327-9.

24. Galante L, Colston KW, MacAuley SJ, et al. Effect of calcitonin on vitamin D metabolism. Nature. 1972;238(5362):271-3.

25. Stevenson JC, Hillyard CJ, MacIntyre I, et al. A physiological role for calcitonin: protection of the maternal skeleton. Lancet. 1979;2(8146):769-70. 
26. Kumar R, Cohen WR, Silva P, et al. Elevated 1,25-dihydroxyvitamin D plasma levels in Invest. 1979;63(2):342-4.

27. Robinson CJ, Spanos E, et al. Role of prolactin in vitamin D metabolism and calcium absorption during lactation in the rat. $J$ Endocrinol. 1982;94(3):443-53.

28. Ajibade DV, Dhawan P, Christakos S. Prolactin: A regulator of the 25-hydroxyvitamin D3 1 alpha hydroxylase gene. J Bone Miner Res. 2009
29. Holick M. Vitamin D deficiency. New Engl Journ Med. 2007; 357(3):266-81.

30. Ross C, Christine L, Taylor Ann L. Committee to Review Dietary Reference Intakes for Vitamin $D$ and Calcium. Institute of Medicine Consensus Report: 2011. 